\title{
Series Solutions of the Anharmonic Motion Equation
} $y^{\prime \prime}+y^{2}=c$

\section{Pisistha Ratanavararaksa ${ }^{1, \mathrm{a}}$ and Mongkol Dejnakarintra ${ }^{2, \mathrm{~b}, *}$ \\ 1 Department of Physics, Faculty of Science, Chulalongkorn University, Thailand 2 The Academy of Science, The Royal Society of Thailand \\ Email: arpisisth@chula.ac.th, bmongkol.d@hotmail.com (Corresponding author)}

\begin{abstract}
Among equations representing motion of a mass or a particle in physics, many of them are anharmonic and consist of one or more nonlinear terms. This article shows how to obtain series solutions of the equation of the form $y^{\prime \prime}(t)+[y(t)]^{2}=c$, which is an anharmonic motion equation. The authors focus the effort on the case with $c=0, y(0)=0, y^{\prime}(0)=$ constant and use of the Taylor's series method; this sets the article apart from those of other authors who did similar works in the past. The series solution obtained under the indicated conditions is of the form $y(t)=\sum_{n=0}^{\infty} a_{3 n} t^{1+3 n}$, which
\end{abstract} does not appear very often in general mathematical functions.

Key words: Series solution, anharmonic motion, Taylor's series.

ENGINEERING JOURNAL Volume 20 Issue 5

Received 20 January 2016

Accepted 19 April 2016

Published 25 November 2016

Online at http://www.engj.org/

DOI:10.4186/ej.2016.20.5.203 


\section{Introduction}

Many researchers have studied anharmonic motions since a long time ago. For example, Helmholtz [1] studied the vibration of the eardrums and set up a mathematical model for the motion as

$$
y^{\prime \prime}(t)+\omega^{2} y(t)=-\alpha[y(t)]^{2} \ldots t>0
$$

and Rayleigh [2] continued the study and specified the initial conditions for Eq. (1) as follows:

$$
y(0)=A \text { and } y^{\prime}(0)=0
$$

This has resulted in the problem being called Rayleigh problem [3]. Other researchers who studied and solved anharmonic motion equations later on are, for example, Shidfar and Sadeghi [4], Chouikha [5, 6], Apostol [7], and Amore and Fernandez [8].

Another anharmonic motion equation similar to Eq. (1) is the Duffing equation, whose general form is

$$
y^{\prime \prime}(t)+\omega^{2} y(t)=-\alpha[y(t)]^{3}
$$

Researchers who studied or solved this equation are, for example, Shidfar and Sadeghi [9], Apostol [7], and Harko et al. [10].

In solving such nonlinear equations as Eqs. (1) and (3), most researchers used the Frobenius method by assuming the solution in terms of a power series of the independent variable $t$, i.e., $y(t)=\sum_{n=0}^{\infty} a_{n} t^{n}$ or that of $\sin (\omega t)$, i.e., $y(t)=\sum_{n=0}^{\infty} a_{n} \sin ^{n}(\omega t)$. Then they determined the recurrence relation among the coefficients $a_{0}, a_{1}, a_{2}, \ldots$ by finding the second derivative of $y(t)$ in power series form and substituted it in the associated equation. Moreover, the researchers mentioned above were only interested in the series solution to the equation that is subject to the initial conditions $y(0)=$ constant, $y^{\prime}(0)=0$, ignoring the solution subject to the conditions $y(0)=0, y^{\prime}(0)=$ constant .

In this paper, the authors present series solutions to the anharmonic motion equation $y^{\prime \prime}(t)+[y(t)]^{2}=c \ldots t>0$, which is a special case of the more general $y^{\prime \prime}(t)+f(y)=g(t)$ [6], but we focus on obtaining the solution by using the Leibnitz-Maclaurin method, which uses the Taylor's (or Maclaurin's) series and determines the coefficients directly from the derivatives of the dependent variable. Moreover, we will consider two cases of the initial conditions for the solutions, one being $y(0)=$ constant, $y^{\prime}(0)=0$ and the other being $y(0)=0 ; y^{\prime}(0)=$ constant, each corresponding to a particular solution. This paper will then serve as a verification for the solutions obtained by previous researchers and at the same time bridge the knowledge gap left by them.

Firstly the authors start with a special case of $c=0$, that is, we start with

$$
y^{\prime \prime}(t)+[y(t)]^{2}=0 \ldots t>0
$$

This equation can be obtained from Eq. (1) by setting $\omega=0$ and $\alpha=1$. Later on we will consider the equation $y^{\prime \prime}(t)+[y(t)]^{2}=c \neq 0 \ldots t>0$.

\section{Series Solution by the Frobenius Method}

The standard form of a series solution for Eq. (4) can be expressed as

$$
y(t)=\sum_{n=0}^{\infty} a_{n} t^{r+n}
$$


By substituting $y(t)$ in Eq. (5) into Eq. (4) we obtain

$$
\sum_{n=0}^{\infty}(r+n)(r+n-1) a_{n} t^{r+n-2}+\left[\sum_{n=0}^{\infty} a_{n} t^{r+n}\right]^{2}=0
$$

which, when expanded in various powers of $t$, yields

$$
\left\{\begin{array}{l}
r(r-1) a_{0} t^{r-2}+(r+1) r a_{1} t^{r-1} \\
+(r+2)(r+1) a_{2} t^{r}+(r+3)(r+2) a_{3} t^{r+1}+\ldots \\
+a_{0}^{2} t^{2 r}+a_{1}^{2} t^{2 r+2}+a_{2}^{2} t^{2 r+4}+a_{3}^{2} t^{2 r+6}+\ldots \\
+2 a_{0} a_{1} t^{2 r+1}+2 a_{0} a_{2} t^{2 r+2}+2 a_{0} a_{3} t^{2 r+3}+\ldots \\
+2 a_{1} a_{2} t^{2 r+3}+2 a_{1} a_{3} t^{2 r+4}+2 a_{1} a_{4} t^{2 r+5}+\ldots \\
+2 a_{2} a_{3} t^{2 r+5}+2 a_{2} a_{4} t^{2 r+6}+2 a_{2} a_{5} t^{2 r+7}+\ldots+\ldots=0
\end{array}\right\}
$$

Considering the term with the lowest power of $t$ in Eq. (7) with the conditions that $2 r>r-2$ (or, equivalently, $r>-2$ ) and $a_{0} \neq 0$, we obtain $r(r-1)=0$, which yields $r=0$ or $r=1$. The case of $r=0$ gives the first solution $y(t)=\sum_{n=0}^{\infty} a_{n} t^{n}$ in accordance with the initial conditions $y(0)=a_{0}$ and $y^{\prime}(0)=a_{1}$. If we specify that $a_{0} \neq 0$ and $a_{1} \neq 0$, the whole series obtained will yield the complete general solution, which consists of two independent component series, one multiplied by $a_{0}$ and the other multiplied by $a_{1}$. On the other hand, the case of $r=1$ gives the second solution $y(t)=\sum_{n=0}^{\infty} a_{n} t^{1+n}$ in accordance with the initial conditions

$$
y(0)=0 \text { and } y^{\prime}(0)=a_{0} \neq 0
$$

This second solution is the special case of the first solution when $a_{0}=0$ and $a_{1}, a_{2}, a_{3}, \ldots$ are replaced with $a_{0}, a_{1}, a_{2}, \ldots$, respectively.

(1) Case of $\underline{r=0}$ : Eq. (7) is reduced to

$$
\left\{\begin{array}{l}
(2 \times 1) a_{2}+(3 \times 2) a_{3} t+(4 \times 3) a_{4} t^{2}+\ldots \\
+a_{0}^{2}+a_{1}^{2} t^{2}+a_{2}^{2} t^{4}+a_{3}^{2} t^{6}+\ldots \\
+2 a_{0} a_{1} t+2 a_{0} a_{2} t^{2}+2 a_{0} a_{3} t^{3}+\ldots \\
+2 a_{1} a_{2} t^{3}+2 a_{1} a_{3} t^{4}+2 a_{1} a_{4} t^{5}+\ldots \\
+2 a_{2} a_{3} t^{5}+2 a_{2} a_{4} t^{6}+2 a_{2} a_{5} t^{7}+\ldots+\ldots=0
\end{array}\right\}
$$

which, when we equate the coefficients of $t^{k}(k=0,1,2, \ldots)$ to zero, yields relationships among various coefficients in Eq. (5) as follows: 


$$
\left\{\begin{array}{l}
a_{2}=-a_{0}^{2} /(2 \times 1) ; a_{3}=-2 a_{0} a_{1} /(3 \times 2) ; \\
a_{4}=-\left(a_{1}^{2}+2 a_{0} a_{2}\right) /(4 \times 3) ; a_{5}=-\left(2 a_{0} a_{3}+2 a_{1} a_{2}\right) /(5 \times 4) ; \ldots ; \\
a_{2 m}=-\left(a_{m-1}^{2}+2 a_{0} a_{2 m-2}+2 a_{1} a_{2 m-3}+\ldots+2 a_{i} a_{j}\right) /[2 m(2 m-1)] \ldots(i+j=2 m-2, i<j) \\
a_{2 m+1}=-2\left(a_{0} a_{2 m-1}+a_{1} a_{2 m-2}+\ldots+a_{i} a_{j}\right) /[(2 m+1)(2 m)] \ldots(i+j=2 m-1, i<j)
\end{array}\right\}
$$

Now if we apply the condition $y^{\prime}(0)=0$ from Eq. (2) by setting $a_{1}=0$, Eq. (10) is reduced to

$$
\left\{\begin{array}{l}
a_{2}=-a_{0}^{2} /(2 \times 1) ; a_{4}=-2 a_{0} a_{2} /(4 \times 3) ; a_{6}=-\left(a_{2}^{2}+2 a_{0} a_{4}\right) /(6 \times 5) ; \ldots \\
a_{4 m}=-2\left(a_{0} a_{4 m-2}+a_{2} a_{4 m-4}+\ldots+a_{i} a_{j}\right) /[4 m(4 m-1)] \ldots(i+j=4 m-2, i<j) \\
a_{4 m+2}=-\left(a_{2 m}^{2}+2 a_{0} a_{4 m}+2 a_{2} a_{4 m-2}+\ldots+2 a_{i} a_{j}\right) /[(4 m+2)(4 m+1)] \ldots(i+j=4 m, i<j) \\
a_{3}=a_{5}=\ldots=a_{2 k+1}=0
\end{array}\right\}
$$

which, when substituted into Eq. (5), gives the first solution to Eq. (4) as

$$
y(t)=a_{0}-\left(a_{0}^{2} / 2 !\right) t^{2}+\left(2 a_{0}^{3} / 4 !\right) t^{4}-\left(10 a_{0}^{4} / 6 !\right) t^{6}+\left(80 a_{0}^{5} / 8 !\right) t^{8}-\ldots
$$

(2) Case of $\underline{r=1}$ : Eq. (7) is reduced to

$$
\left\{\begin{array}{l}
(2 \times 1) a_{1}+(3 \times 2) a_{2} t+(4 \times 3) a_{3} t^{2}+(5 \times 4) a_{4} t^{3}+\ldots \\
+a_{0}^{2} t^{2}+a_{1}^{2} t^{4}+a_{2}^{2} t^{6}+a_{3}^{2} t^{8}+\ldots \\
+2 a_{0} a_{1} t^{3}+2 a_{0} a_{2} t^{4}+2 a_{0} a_{3} t^{5}+\ldots \\
+2 a_{1} a_{2} t^{5}+2 a_{1} a_{3} t^{6}+2 a_{1} a_{4} t^{7}+\ldots \\
+2 a_{2} a_{3} t^{7}+2 a_{2} a_{4} t^{8}+2 a_{2} a_{5} t^{9}+\ldots+\ldots=0
\end{array}\right\}
$$

which, when we equate the coefficients of $t^{k}(k=0,1,2, \ldots)$ to zero, yields

$$
\left\{\begin{array}{l}
a_{1}=0 ; a_{2}=0 ; a_{3}=-a_{0}^{2} /(4 \times 3) ; a_{4}=-2 a_{0} a_{1} /(5 \times 4)=0 ; a_{5}=-\left(a_{1}^{2}+2 a_{0} a_{2}\right) /(6 \times 5)=0 ; \\
a_{6}=-2\left(a_{0} a_{3}+a_{1} a_{2}\right) /(7 \times 6)=-2 a_{0} a_{3} /(7 \times 6)=20 a_{0}^{3} /(7 !) ; \ldots \\
a_{2 m}=\frac{-2\left(a_{0} a_{2 m-3}+a_{1} a_{2 m-4}+\ldots+a_{i} a_{j}\right)}{(2 m+1)(2 m)} \ldots(i+j=2 m-3, i<j) \\
a_{2 m+1}=\frac{-\left(a_{m-1}^{2}+2 a_{0} a_{2 m-2}+2 a_{1} a_{2 m-3}+\ldots+2 a_{i} a_{j}\right)}{(2 m+2)(2 m+1)} \ldots(i+j=2 m-2, i<j)
\end{array}\right\}
$$

From Eq. (14) we find that only $a_{0}, a_{3}, a_{6}, a_{9}, \ldots$ or $a_{3 k}(k=0,1,2, \ldots)$ are not equal to zero. And when we substitute these coefficients into Eq. (5) with $r=1$, we find the second solution to Eq. (4) as

$$
y(t)=a_{0} t-\left(2 a_{0}^{2} / 4 !\right) t^{4}+\left(20 a_{0}^{3} / 7 !\right) t^{7}-\left(600 a_{0}^{4} / 10 !\right) t^{10}+\ldots
$$

which apparently has not been shown by other authors. 
We can show that this second solution, with $r=1$, is the special case of the general solution with $r=0$ when $a_{0}=0$ as follows:

In Eq. (10), if we set $a_{0}=0$, we obtain for the first few terms of the series

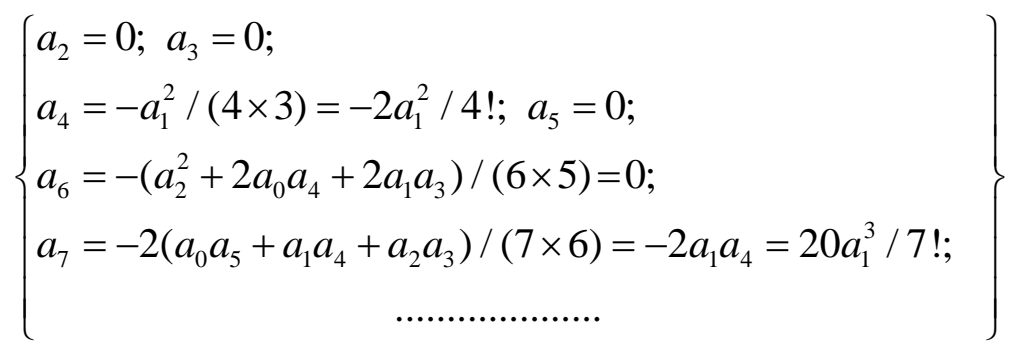

and the solution becomes $y(t)=\sum_{n=0}^{\infty} a_{n} t^{n}=a_{1} t-\left(2 a_{1}^{2} / 4 !\right) t^{4}+\left(20 a_{1}^{3} / 7 !\right) t^{7}-\ldots$, which, when $a_{1}$ is replaced with $a_{0}$, verifies the second solution, with $r=1$, in Eq. (15).

\section{Series Solution by the Leibnitz-Maclaurin Method}

Since the value of $r$ in Eq. (7) is 0 or 1, Eq. (5) as solution to Eq. (4) will be a Taylor's (or Maclaurin's) series, whose general form is

$$
y(t)=y(0)+y^{\prime}(0) t+\left[\frac{y^{\prime \prime}(0)}{2 !}\right] t^{2}+\left[\frac{y^{\prime \prime \prime}(0)}{3 !}\right] t^{3}+\left[\frac{y^{(4)}(0)}{4 !}\right] t^{4}+\ldots
$$

where $y^{\prime \prime}(0), y^{\prime \prime \prime}(0), y^{(4)}(0), \ldots, y^{(10)}(0)$ can be obtained by successively taking derivatives of Eq. (4) in accordance with the Leibnitz-Maclaurin method as follows:

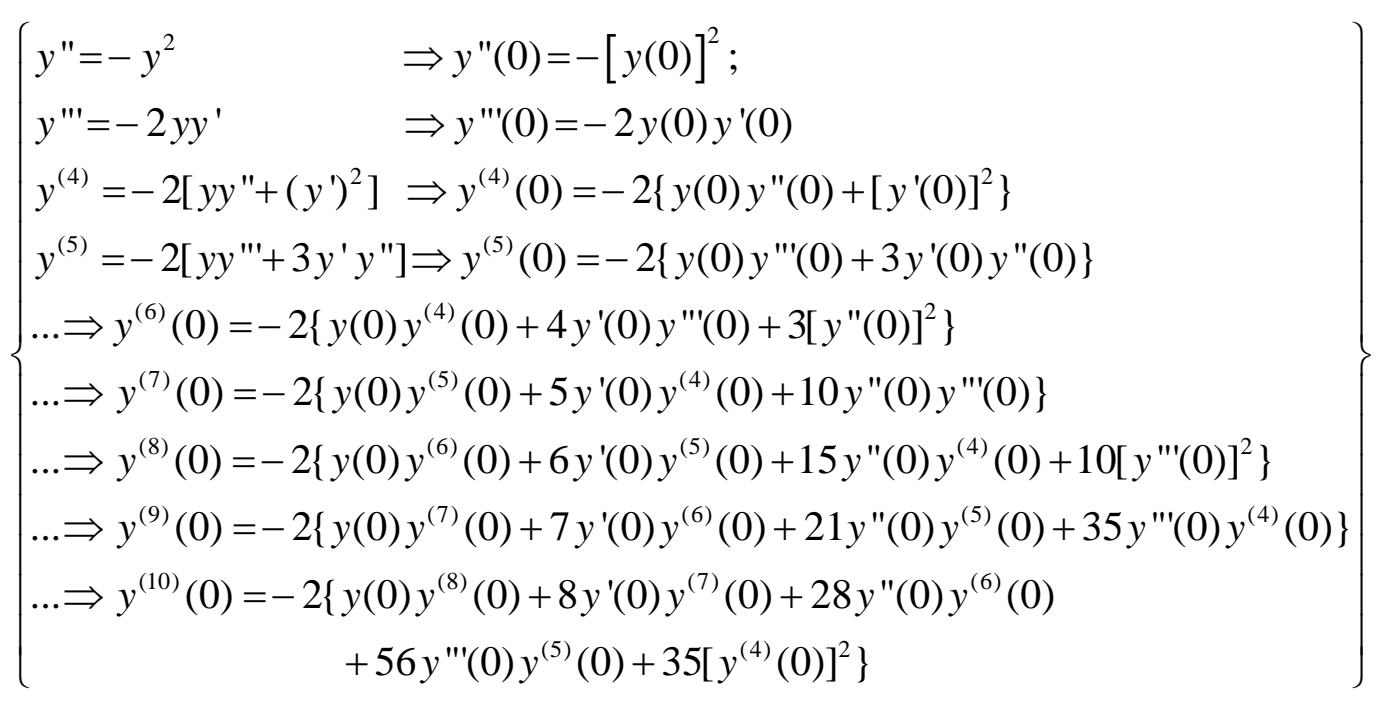

In general we obtain the following relationships: 


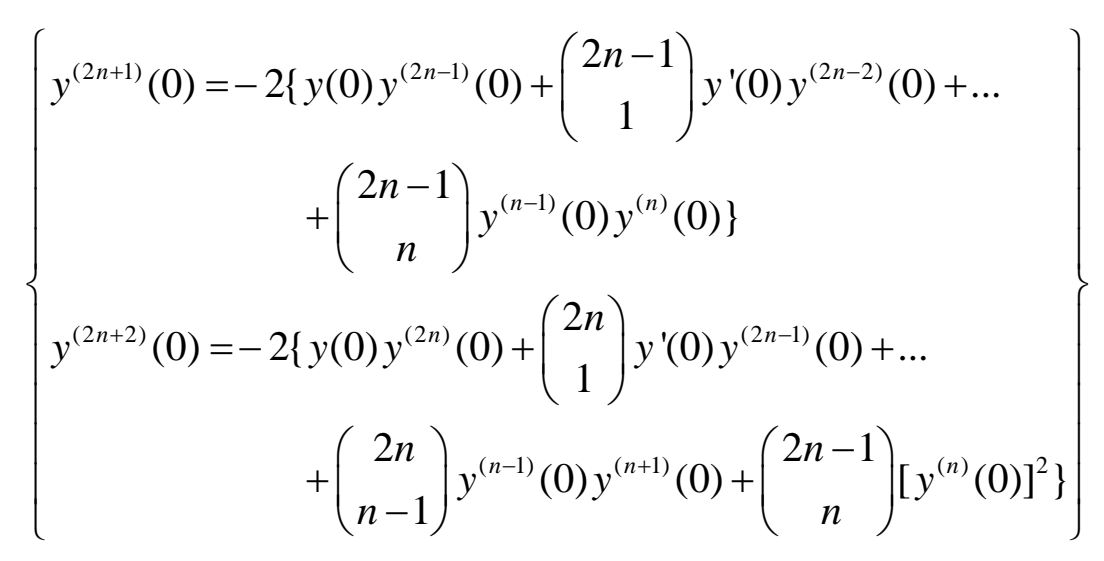

where $n \geq 2$ and $\left(\begin{array}{l}m \\ k\end{array}\right) \triangleq \frac{m !}{k !(m-k) !}$ is the binomial coefficient.

(1) Case of $\underline{y(0)=a} \underline{\text { and }} \underline{y^{\prime}(0)=0}$ : Eq. (17) yields

$$
\left\{\begin{array}{l}
y^{\prime \prime \prime}(0)=y^{(5)}(0)=y^{(7)}(0)=\ldots=y^{(2 m+1)}(0)=0 \ldots(m=1,2,3, \ldots) \\
y^{\prime \prime}(0)=-a^{2} ; y^{(4)}(0)=2 a^{3} ; y^{(6)}(0)=-10 a^{4} ; y^{(8)}(0)=80 a^{5} ; \ldots
\end{array}\right\}
$$

and Eq. (16) yields the first solution as

$$
y(t)=a-\left(a^{2} / 2 !\right) t^{2}+\left(2 a^{3} / 4 !\right) t^{4}-\left(10 a^{4} / 6 !\right) t^{6}+\left(80 a^{5} / 8 !\right) t^{8}-\ldots
$$

which is the same as Eq. (12) in the last section.

(2) $\underline{\text { Case of }} \underline{y(0)=0} \underline{\text { and }} \underline{y^{\prime}(0)=b}$ : Eq. (17) yields

$$
\left\{\begin{array}{l}
y^{\prime \prime}(0)=0 ; y^{\prime \prime}(0)=0 ; y^{(5)}(0)=0 ; y^{(6)}(0)=0 ; y^{(8)}(0)=0 ; y^{(9)}(0)=0 ; \ldots \\
y^{(4)}(0)=-2 b^{2} ; y^{(7)}(0)=20 b^{3} ; y^{(10)}(0)=-600 b^{4} ; \ldots
\end{array}\right\}
$$

and Eq. (16) yields the second solution as

$$
y(t)=b t-\left(2 b^{2} / 4 !\right) t^{4}+\left(20 b^{3} / 7 !\right) t^{7}-\left(600 b^{4} / 10 !\right) t^{10}+\ldots
$$

which is the same as Eq. (15) in the last section.

\section{Comparison Between the Frobenius Method and the Leibnitz-Maclaurin Method}

Comparing the Frobenius method and the Leibnitz-Maclaurin method for series solutions to the same differential equation, we can see that the mathematical procedure of the first method is easier because in finding $y^{\prime \prime}(t)$ the differentiations are done on the power terms $t^{n}$ of the independent variable $t$ only, but in the second method the differentiations needed for finding $y^{(n)}(t),(n>2)$ are done on product terms involving the dependent variable $y(t)$ and its derivatives $y^{\prime}(t), y^{\prime \prime}(t), \ldots, y^{(n-2)}(t)$. However, in the first method the coefficients $a_{n}$ of the power series are not readily known, i.e., not until like power terms have been compared, whereas in the second method $a_{n}$ is readily obtained from $y^{(n)}(0) / n$ !. 


\section{Solution to the Rayleigh Problem by the Leibnitz-Maclaurin Method}

In this section we will solve Eq. (1) subject to the initial conditions in Eq. (2) by using the Taylor's series as in the section before the last. For convenience, we transform Eq. (1) by changing the independent and dependent variables as follows:

For the independent variable, we set $s=\sqrt{\alpha} t \Rightarrow \frac{d(.)}{d t}=\sqrt{\alpha} \frac{d(.)}{d s}$ so that Eq. (1) becomes

$$
y^{\prime \prime}(s)+[y(s)]^{2}+\left(\frac{\omega^{2}}{\alpha}\right) y(s)=0 \Rightarrow y^{\prime \prime}(s)+\left[y(s)+\frac{\omega^{2}}{2 \alpha}\right]^{2}=\frac{\omega^{4}}{4 \alpha^{2}}
$$

which, when we set the new dependent variable $u(s)=y(s)+\frac{\omega^{2}}{2 \alpha}$, yields

$$
u^{\prime \prime}(s)+[u(s)]^{2}=c
$$

where $c=\omega^{4} /\left(4 \alpha^{2}\right)$ and the corresponding initial conditions are

$$
u(0)=A+\sqrt{c}=A+\omega^{2} /(2 \alpha) ; u^{\prime}(0)=0
$$

The results above show that Eq. (4) is a special case of Eq. (23) with $c=0$.

By successively taking derivative of Eq. (23) with respect to $s$, we obtain

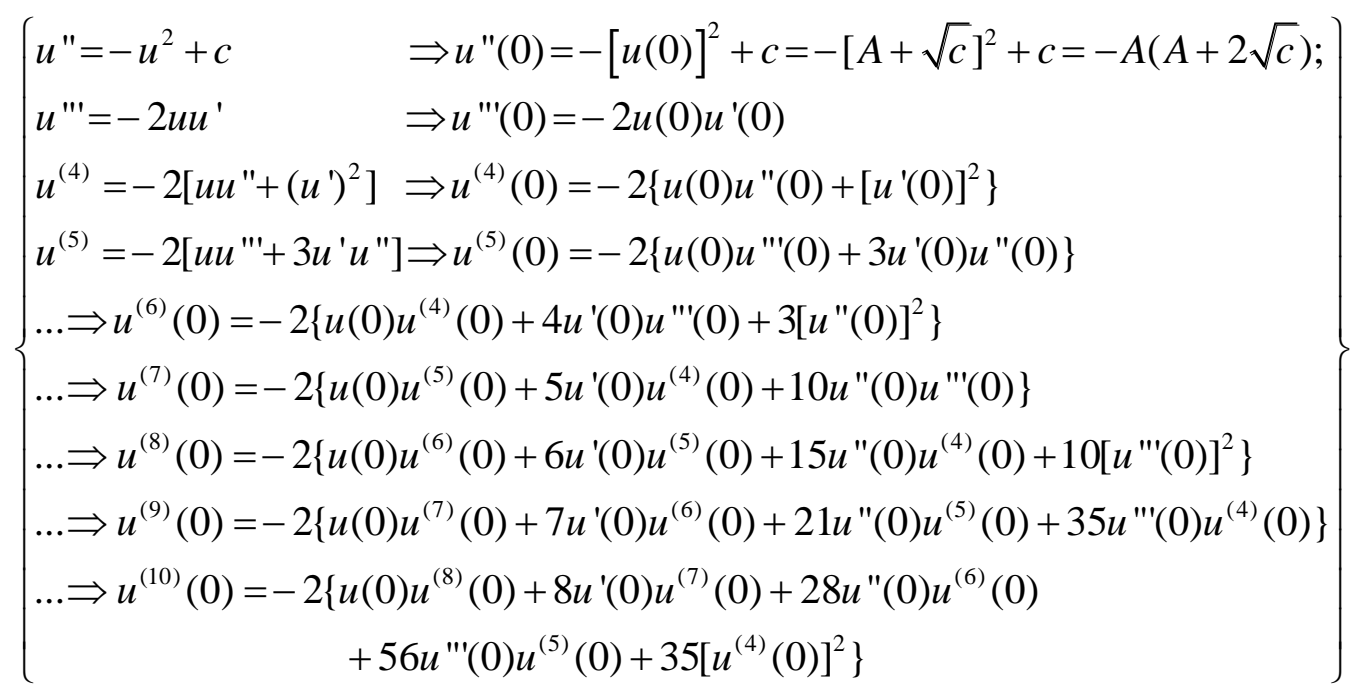

which has the same form as Eq. (17) in the section before the last but has a different expression for $u$ "(0) from that for $y^{\prime \prime}(0)$ in Eq. (17). In general we obtain 


$$
\left\{\begin{array}{r}
u^{(2 n+1)}(0)=-2\left\{u(0) u^{(2 n-1)}(0)+\left(\begin{array}{c}
2 n-1 \\
1
\end{array}\right) u^{\prime}(0) u^{(2 n-2)}(0)+\ldots\right. \\
\left.+\left(\begin{array}{c}
2 n-1 \\
n
\end{array}\right) u^{(n-1)}(0) u^{(n)}(0)\right\} \\
u^{(2 n+2)}(0)=-2\left\{u(0) u^{(2 n)}(0)+\left(\begin{array}{c}
2 n \\
1
\end{array}\right) u^{\prime}(0) u^{(2 n-1)}(0)+\ldots\right. \\
\left.+\left(\begin{array}{c}
2 n \\
n-1
\end{array}\right) u^{(n-1)}(0) u^{(n+1)}(0)+\left(\begin{array}{c}
2 n-1 \\
n
\end{array}\right)\left[u^{(n)}(0)\right]^{2}\right\}
\end{array}\right\}
$$

similar to those in Eq. (18).

When we set $u^{\prime}(0)=0$ in Eq. (25), we get

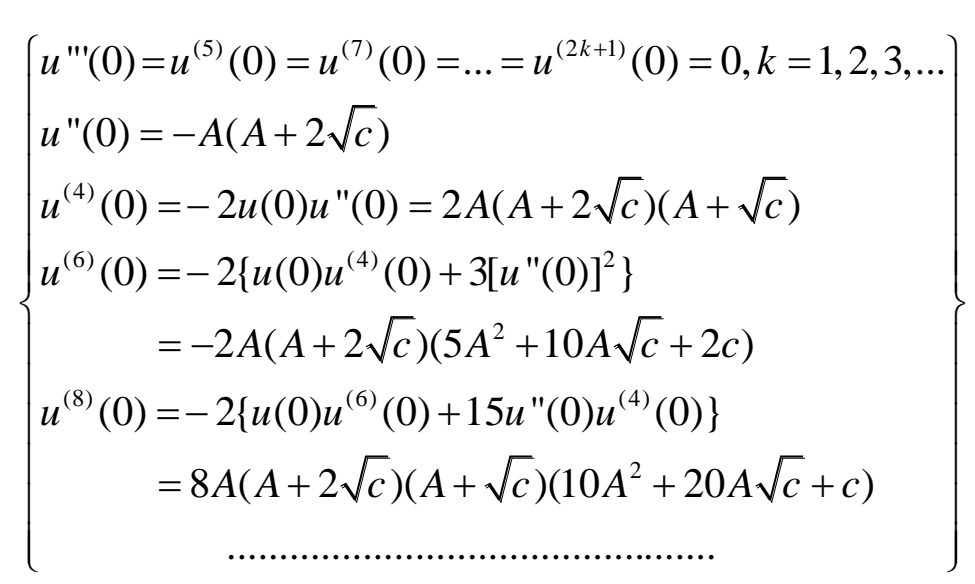

which, when substituted into the Taylor's series for $u(s)$, namely,

$$
u(s)=u(0)+u^{\prime}(0) s+\left[\frac{u^{\prime \prime}(0)}{2 !}\right] s^{2}+\left[\frac{u^{\prime \prime \prime}(0)}{3 !}\right] s^{3}+\left[\frac{u^{(4)}(0)}{4 !}\right] u^{4}+\ldots
$$

results in

$$
u(s)=\left\{\begin{array}{l}
(A+\sqrt{c})-A\left(\left[\frac{A+2 \sqrt{c}}{2 !}\right] s^{2}-2\left[\frac{(A+2 \sqrt{c})(A+\sqrt{c})}{4 !}\right] s^{4}\right. \\
\left.+2\left[\frac{(A+2 \sqrt{c})\left(5 A^{2}+10 A \sqrt{c}+2 c\right)}{4 !}\right] s^{6}-\ldots\right)
\end{array}\right\}
$$

If we set $s=\sqrt{\alpha} t$ and $y(s)=u(s)-\sqrt{c}$, we obtain the solution to the Rayleigh problem as

$$
y(t)=\left\{\begin{array}{l}
A\left(1-\left[\frac{A+2 \sqrt{c}}{2 !}\right] \alpha t^{2}+2\left[\frac{(A+2 \sqrt{c})(A+\sqrt{c})}{4 !}\right] \alpha^{2} t^{4}\right. \\
\left.-2\left[\frac{(A+2 \sqrt{c})\left(5 A^{2}+10 A \sqrt{c}+2 c\right)}{4 !}\right] \alpha^{3} t^{6}+\ldots\right)
\end{array}\right\}
$$


which is the same as that obtained by Shidfar and Sadeghi [4] using the Frobenius method.

In Eq. (29) if we set $c=0, \alpha=1$, and $A=a_{0}$, we will get the same expression for $y(t)$ as the solution to $y^{\prime \prime}+y^{2}=0, y(0)=a_{0}, y^{\prime}(0)=0$ obtained in Eq. (12) and (20).

\section{Convergence of the Solution}

The solution to $u^{\prime \prime}(s)+[u(s)]^{2}=c$ given in Eq. (28) is convergent for all values of $s$ since this equation does not have a singular point, and every point $s$ in the $s$ - $u$ plane is an ordinary point of the equation. This is in accordance with the explanation given by Shidfar and Sadeghi [4]. By using the same reasoning, we can conclude that the solutions to $y^{\prime \prime}(t)+[y(t)]^{2}=0$ given in Eq. (12), (15), (20), and (22) are convergent for all values of $t$.

\section{Other Forms of Solution}

The equations $y^{\prime \prime}(t)+[y(t)]^{2}=0$ and $u^{\prime \prime}(s)+[u(s)]^{2}=c$ are special cases of the equation of the form $y^{\prime \prime}(t)=f(y)$, which is called second-order autonomous equation (see, for example, Polyanin and Zaitsev [11]) and has a solution in the integral form as follows:

$$
\int \frac{d y}{\sqrt{C_{1}+2 \int f(y) d y}}= \pm t+C_{2}
$$

where $C_{1}, C_{2}$ are arbitrary constants.

Apostol [7] showed that a solution to the Rayleigh problem in Eqs. (1) and (2), which can be transformed into $u^{\prime \prime}(s)+[u(s)]^{2}=c$, can be expressed as the elliptic integral of the first kind $F(\varphi, k)$ (see, for example, Spiegel and Liu [12]):

$$
F(\varphi, k)=\int_{0}^{\varphi} \frac{d \theta}{\sqrt{1-k^{2} \sin ^{2} \theta}} \ldots(0<k<1)
$$

whereas Harko et al. [10] showed that a solution to $y^{\prime \prime}(t)+[y(t)]^{n}=0 \ldots(n \neq-1)$, which has $y^{\prime \prime}(t)+[y(t)]^{2}=0$ as a special case, can be expressed as the hypergeometric function ${ }_{2} F_{1}(a, b ; c ; d)$ (see, for example, Spiegel and Liu [12]).

However, all of the solutions mentioned in this section are in the form of $t=F(y)$, which is the reverse of the relationship $y=G(t)$ in the series solutions given in the earlier sections of this paper.

\section{Sample Applications}

In this section, the authors will show two practical applications of the anharmonic motion equation $y^{\prime \prime}(t)+[y(t)]^{2}=c$, one in physics and the other in electrical engineering.

Figure 1 shows a spring-mass system for which $k$ is the spring constant, $m$ the mass, $y$ the displacement of the mass from the equilibrium position, and $F$ the applied force. Assuming here that the spring is nonlinear, with its restoring force being $k y^{2}$, we can write the equation of motion as

$$
m \frac{d^{2} y}{d t^{2}}+k y^{2}=F \Rightarrow y^{\prime \prime}(t)+\alpha^{2}[y(t)]^{2}=\beta F
$$


where $\alpha^{2}=k / m$ and $\beta=1 / m$. Next, if we change the independent variable from $t$ to $\tau=\alpha t$ and let $F$ be a constant, Eq. (32) will be transformed into

$$
y^{\prime \prime}(\tau)+[y(\tau)]^{2}=c
$$

where $c=\beta F / \alpha^{2}$. Equation (33) is in the form of the anharmonic equation of this paper.

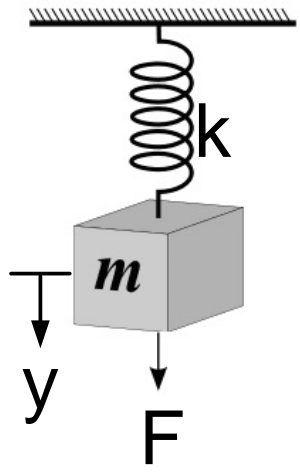

Fig. 1. A spring-mass system

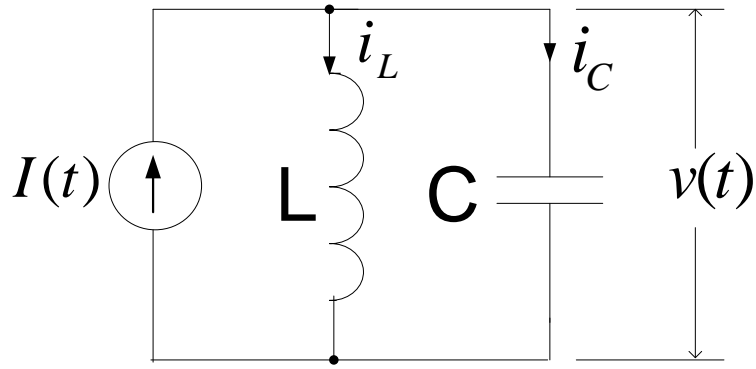

Fig. 2. A parallel LC circuit

Figure 2 shows a parallel electric circuit consisting of a current source $I(t)$, an inductor $L$, and a capacitor $C$. If we let $i_{L}(t)$ and $i_{C}(t)$ be the inductor current and the capacitor current, respectively, with $v(t)$ being the voltage across the parallel connection, then the Kirchhoff Current Law will yield

$$
i_{C}(t)+i_{L}(t)=I(t) \Rightarrow C \frac{d v(t)}{d t}+\left(\frac{1}{L}\right) \int_{0}^{t} v(t) d t+i_{L}(0)=I(t)
$$

By differentiating Eq. (34) once with respect to $t$ and rearrange the resulting equation, we obtain

$$
v^{\prime \prime}(t)+\left(\frac{1}{C}\right) \frac{v(t)}{L}=\frac{I^{\prime}(t)}{C}
$$

If the inductance $L$ varies inversely with $v(t)$, i.e., $L=k / v(t)$, and $I^{\prime}(t)$ is constant, i.e., $I(t)=K t$, then Eq. (35) becomes

$$
v^{\prime \prime}(t)+\alpha^{2}[v(t)]^{2}=\beta K
$$

where $\alpha^{2}=1 /(k C)$ and $\beta=1 / C$. Equation (36) has the same form as Eq. (32) and can also be transformed into the anharmonic equation, Eq. (33), of our interest.

\section{Discussion}

The equation $y^{\prime \prime}(t)+[y(t)]^{2}=0$ is similar to $y^{\prime \prime}(t)+y(t)=0$ but the two are different in that the former is nonlinear whereas the latter is linear. From the physical viewpoint, the former equation represents a spring-mass system in which the spring gives a restoring force proportional to the square of the displacement $\left(y^{2}\right)$ so that the motion of the mass is anharmonic. However, the latter equation represents a spring-mass system in which the spring gives a restoring force proportional to the displacement $(y)$ so that the motion of the mass is harmonic. From the mathematical viewpoint, the two 
particular solutions of the former equation cannot form a linear combination to be used as a new solution, but those for the latter equation can do so.

Although the series solution to the equation $y^{\prime \prime}(t)+f(y)=g(t)$ has been studied by some researchers (see [6], for example), the equation $y^{\prime \prime}(t)+[y(t)]^{2}=0$ with the initial conditions $y(0)=0$ and $y^{\prime}(0)=a_{0} \neq 0$ has not been explicitly solved before. The solution given in Eq. (15) and (22) in this paper is an oddly-looking power series compared with other series that represent such ordinary mathematical functions as $\exp (t), \sin (t), \cos (t)$. Its special feature is that the power of the variable $t$ increases by 3 from term to term (the solution is of the form $\left.y(t)=\sum_{n=0}^{\infty} a_{3 n} t^{1+3 n}\right)$. So the first author of this paper, who had a long career as an official of Chulalongkorn University, Thailand, proposed that it be called the Chula series in honor of the University.

\section{Conclusion}

This paper shows how to obtain the series solutions to the equation $y^{\prime \prime}(t)+[y(t)]^{2}=c$, focusing on the special case of $c=0, y(0)=0, y^{\prime}(0)=$ constant and on the Taylor's series method. This bridges the knowledge gap left by the works of other authors. The power series solution in the form $y(t)=\sum_{n=0}^{\infty} a_{3 n} t^{1+3 n}$ appears infrequently in any mathematical textbook in comparison with such well-known functions as $\exp (t), \sin (t)$, and $\cos (t)$.

\section{Acknowledgment}

The second author and the wife of the late first author wish to thank Prof. Dr. Yongwimol Lenbury of The Academy of Science, The Royal Society of Thailand, for her useful comments for improvement of the first draft of this paper.

\section{References}

[1] H. L. F. Helmholtz, On the Sensation of Tone. New York: Dover, 1945.

[2] J. W. S. Rayleigh, Theory of Sound, vol. 1. New York: Dover, 1945.

[3] J. R. Usher and V. A. Nye, "Further observation on the anharmobic motion equation," Int. J. Math. Educ. Sci. Technol., vol. 20, no. 3, pp. 399-406, 1989.

[4] A. A. Shidfar and A. A. Sadeghi, "Some series solutions of the anharmonic motion equation," J. Math. Anal. Appl., vol. 120, pp. 488-493, 1986.

[5] A. R. Chouikha, "Series solutions of some anharmonic motion equations," J. Math. Anal. Appl., vol. 272, pp. 79-88, 2002.

[6] A. R. Chouikha, "Solutions series for some non-harmonic motion equations," Electron. J. Diff. Eqs, vol. 10, pp. 115-122, 2003.

[7] B. F. Apostol," On anharmonic oscillators," Rom. Journ. Phys., vol. 50, no. 7-8, pp. 915-918, 2005.

[8] P. Amore and F. M. Fernandez, "Exact and approximate expressions for the period of anharmonic oscillators," Eur. J. Phys., vol. 26, no. 4, pp. 589-601, 2005.

[9] A. A. Shidfar and A. A. Sadeghi, "The periodic solutions of certain non-linear oscillators," Appl. Math. Lett., vol. 3, no. 4, pp. 21-24, 1990.

[10] T. Harko, F. S. N. Lobo, and M. K. Mak, "Integrability cases for the anharmonic oscillator equation," Journal of Pure and Applied Mathematics: Advances and Applications, vol. 10, no. 1, pp. 115-129, 2013.

[11] A. D. Polyanin and V. F. Zaitsev, Handbook of Exact Solutions for Ordinary Differential Equations. Boca Raton: Chapman \& Hall/CRC, 2003.

[12] M. R. Spiegel and J. Liu, Mathematical Handbook of Formulas and Tables, Schaum's Outline Series, 2nd ed. New York: McGraw-Hill, 1999. 